Der Ansicht von Krausch und Kox, daß Hilfsmittel wie beispielsweise der von ihnen angeführte dynamische Score von René Chang et al. aus Riyadh einer „objektiven Entscheidungsfindung dienen könnten“, muß für die klinische Entscheidungsfindung entschieden widersprochen werden. Dies wurde von Chang et al. auch nicht intendiert, die ausdrücklich betonen: „We are not proposing that our predictive model ... is ready to be used to support clinical decision-making “ $[1]$. Zu einer gleichlautenden Aussage kommt auch die zweite europäische Konsensus-Konferenz zur Intensivmedizin im Jahre 1993, die den Einsatz von Scores zur Quantifizierung des Krankheitsschweregrades zum Thema hatte:

„Die Scoring-Systeme wurden entwickelt für die Vorhersage innerhalb großer Gruppen; daher dürfen sie nur für Patientengruppen verwendet werden. Die Vorhersage in individuellen Fällen ist entscheidend eingeschränkt, wenn nicht gar unmöglich" [2].

\section{Literatur}

1. Chang RWS, Jacobs S, Lee B, Pace N (1988) Predicting deaths among intensive care unit patients. Crit Care Med 16:34-42

2. Zweite Europäische Konsensus-Konferenz für Intensivmedizin (Hrsg) (1995) Outcome-Vorhersage in der Intensivmedizin. Anästh Intensivmed 36:154-160

Th. Erb' $\cdot$ Ch. Kern ${ }^{1}$ - F.J. Frei ${ }^{2} \cdot{ }^{1}$ Departement Anaesthesie, Universitätskliniken Kantonsspital Basel ${ }^{2}$ Departement Anaesthesie Universitätskinderklinik Basel

\title{
Die Kehlkopfmaske - ein wertvolles Instrument bei erschwerter kindlicher Intubation
}

\section{Bemerkungen zur Arbeit von R. Ofer und H. Dworzak Anaesthesist (1996) 45:268-270}

er vorgestellte Fallbericht ist ein weiteres Beispiel dafür, daß der Kehlkopfmaske eine eigenständige Bedeutung im Management des schwierigen Atemwegs zukommt, was dazu geführt hat, daß dieses Hilfsmittel Eingang in entsprechende Algorithmen gefunden hat [1].

Bezüglich dem spezifischen Management bei kindlichen mandibulofazialen Mißbildungen sind wir mit den Autoren der Auffassung, daß die fiberoptische Intubation die Methode der Wahl ist. Diese Technik kann aufgrund verschiedener Umstände (teure Ausrüstung, spezielle Handhabung) nur an wenigen Orten zur Verfügung stehen. Das Management des schwierigen Atemwegs beim Kind mit Hilfe des Fiberbronchoskops hat in jüngster Zeit Verbesserungen erfahren. Die Anwendung verschiedener Fiberskopgrößen (z.B. $2,1 \mathrm{~mm}, 3,7 \mathrm{~mm}$, und $4,9 \mathrm{~mm}$ ) erlaubt die Intubation mit Tuben mit einem Innendurchmesser 2,5-8 $\mathrm{mm}$ [2]. Speziell entwickelte Endoskopiemasken ermöglichen die kontinuierliche Verabreichung von $100 \%$ Sauerstoff unter Spontanatmung oder Beatmung während der fiberoptischen Intubation [2], dies vor dem Hintergrund der speziellen Hypoxiegefährdung des pädiatrischen Kollektivs [3, 4].

Die im Fallbericht geschilderte Vorgehensweise (mehrmalige konventionelle Versuche, blind nasale Versuche) birgt die Gefahr von Ödemen und Blutungen der Schleimhaut in sich, die bei den engen kindlichen Verhältnissen rasch zu einer potentiell gefährlichen Atemwegsobstruktion führen kann. U.E. hätte deshalb dieses 6jährige Kind an eine Institution überwiesen werden sollen, die eine elektive fiberoptische Intubation durchführen kann.

Trotz glücklichem Verlauf darf im vorliegenden Fall nicht in Vergessenheit geraten, daß eine nasale Intubation geplant war, die in den Händen eines entsprechend ausgerüsteten und ausgebildeten Teams unter Zuhilfenahme der Fiberoptik mit hoher Sicherheit erfolgreich durchgeführt werden kann.

\section{Literatur}

1. Benumof $J(1996)$ Laryngeal mask airway and the ASA difficult airway algorhithm.

Anesthesiology 84:686-699

2. Frei FJ, áWengen DF, Rutishauser M,Ummenhofer W (1995) The airway endoskopy mask: useful device for fibreoptic evaluation and intubation of the paediatric airway.

Paed Anaesth 5:319-324

3. Coté JC, Goldstein EA, Coté MA, Hoaglin DC, Ryan JF (1988) A single-blind study of pulse oximetry in children. Anesthesiology 68:184-188

4. Patel R, Lenczyk M, Hannallah RS, McGill WA (1994) Age and the onset of desaturation in apnoeic children. Can J Anaesth 41:771-774

Dr.Th. Erb

Departement Anaesthesie, Universitätskliniken Kantonsspital Basel, Spitalstraße 21, CH-4031 Basel 